\title{
Novelty, coherence, and Mendeleev's periodic table
}

\begin{abstract}
Predictivism is the view that successful predictions of 'novel' evidence carry more confirmational weight than accommodations of already known evidence. Novelty, in this context, has traditionally been conceived of as temporal novelty. However temporal predictivism has been criticized for lacking a rationale: why should the time order of theory and evidence matter? Instead, it has been proposed, novelty should be construed in terms of use-novelty, according to which evidence is novel if it was not used in the construction of a theory. Only if evidence is use-novel can it fully support the theory entailing it. As I point out in this paper, the writings of the most influential proponent of use-novelty contain a weaker and a stronger version of use-novelty. However both versions, I argue, are problematic. With regard to the appraisal of Mendeleev' periodic table, the most contentious historical case in the predictivism debate, I argue that temporal predictivism is indeed supported, although in ways not previously appreciated. On the basis of this case, I argue for a form of so-called symptomatic predictivism according to which temporally novel predictions carry more confirmational weight only insofar as they reveal the coherence of facts as identified by the theory to be real.
\end{abstract}

Keywords: novel prediction, use-novelty, coherence, Dmitri Mendeleev, periodic table

\section{Introduction}

Intuitions can be strong and nevertheless deceptive. The status of predictions in theory assessment appears to confirm this. Most of us share the intuition that a theory's successful prediction of an unexpected physical effect should count strongly in favour of that theory and in fact more so than that theory's explanations or 'accommodations' of known facts. It is also said that there exists a confirmatory asymmetry between predictions and accommodations, in favour of the former. But what is the rationale for that intuition? And what is the historical evidence for this intuition? According to Worrall $(1985,2002,2005)$ the answers to both of these questions is "there is none". Worrall instead proposes that in science theories are assessed on the basis of the criterion of use-novelty: evidence is novel not only when it is novel in the temporal sense, but also if it was not used in the construction of the theory that entails it. The use-novelty criterion is much more permissive than temporal novelty: evidence which does not count as novel on the temporal novelty criterion may indeed count as novel on the use-novelty criterion. In contrast to the temporal novelty criterion, says Worrall, the use-novelty criterion comes equipped with a rationale: evidence that was used in the construction of a theory, which that same theory entails, should not count in that theory's favour since the theory's 
accommodation of the evidence was guaranteed by the way in which the theory was constructed. ${ }^{1}$ Temporal predictivism, i.e. the view that phenomena discovered after the theory predicted them count more than phenomena that were known at the time the theory was proposed, Worrall points out, lacks a rationale. Why should it matter whether or not the theory or the evidence which the theory entails was known first?

Besides criticizing temporal predictivism for the lack of rationale, Worrall has provided two in-depth historical studies (one with his collaborator Eric Scerri) where he seeks to show that there is no historical evidence for the view that scientists value temporally novel predictions more than the explanation of already known facts. These cases concern Fresnel's white spot prediction in 1819 (Worrall, 1989) and Mendeleev's prediction of new chemical elements in the late nineteenth century (Scerri \& Worrall, 2001) ${ }^{2}$. The first case is uncontroversial. Worrall skillfully and convincingly shows that, contrary to all prior expectations, our intuitions at least in this case are mistaken. Poisson, a member of the jury assessing Fresnel's work for the prestigious award of the French Academy, derived an apparent absurd consequence, which had not been recognized by Fresnel himself: Fresnel's theory predicted that light emanating from a small hole shone onto an opaque disc would result in a small bright spot at the center of the disc's shadow. But Worrall finds no evidence whatsoever in the lengthy reports of the Academy that this prima facie impressive prediction had any more import for the prize judges than Fresnel's explanation of straight edge diffraction patterns that had been known for decades before Fresnel had proposed his theory. If anything, more positive emphasis was laid upon Fresnel's explanation than on his successful predictions ${ }^{3}$. The assessment of the status of Mendeleev's predictions of new chemical elements is much more controversial. The clarification of this controversy will be one of the main aims of this paper.

This is how I proceed. In Section 2, I critically discuss both a weak and a strong version of Worrall's account of use-novelty, which his writings contain side-by-side, and which has led to considerable confusion in the literature. In Section 3 I assess the historical evidence for and against temporal predictivism in the appraisal of Mendeleev's periodic table. In Section 4, I introduce Hitchcock and Sober's (2004) concept of localsymptomatic predictivism. With regard to Mendeleev's periodic table I then argue for a specific form of symptomatic predictivism, according to which successful predictions are indicators for the theory having correctly identified a coherence of facts. In the conclusion of this paper (section 5) I propose that a local-

\footnotetext{
${ }^{1}$ Worrall (1989 148-9) explicitly refers to Popper (1959) for having first articulated this intuition. The idea that hypotheses should pass severe tests has more recently been interpreted in terms of error statistics (Mayo, 1991).

${ }^{2}$ See also various case studies by the historian Stephen Brush. There is furthermore a set of non-historical examples that has been circulated, all in in favour of temporal predictivism (Lange, 2001; Maher, 1988; White, 2003). Harker (2006) argues convincingly that these examples do not bear on the predictivism debate, for their persuasiveness depends on the belief that the predicted events in question are unpredictable in principle (such as the outcome of a lottery). If given evidence that, under certain circumstances (e.g. a rigged lottery, an unfair coin), prima facie unpredictable events have indeed been genuinely predicted, we are certainly bound to be impressed. However predictions in science do not have that character. Although certain predictions might be surprising, they are generally not predictions of prima facie unpredictable events.

${ }^{3}$ In fact Fresnel's theory made another surprising prediction that was confirmed. It predicted a 'black spot' of total darkness at certain distances beyond (and in line of) a hole in an opaque screen on which light is shone.
} 
symptomatic predictivism might be the most plausible theory of the status of predictions in the appraisal theories in general.

\section{Weak and strong use-novelty}

Worrall's writings (both old and new) contain two unappreciated versions of the use-novelty criterion. In the weaker version,

Weak UN: Evidence $e$ counts as novel with regard to a theory T entailing e, if $e$ was not used in the construction of T. Evidence that is use-novel with regard to T supports T.

In this version, in order to judge whether or not a given piece of evidence supports the theory that entails this evidence, "we have to look at how the theory was constructed" (Worrall 1978, p. 313). In other words, Worrall believes the context of discovery to directly bear on the context of justification. Furthermore, on Worrall's view, it is a mistake to treat the "downgrading of ad hoc explanations and the apparent upgrading of genuine predictions as two separate methodological phenomena". "At root", Worrall continues", they are "the same phenomena" (1989, p. 148). We can therefore formulate a criterion for what it is for a theory to be ad hoc, which is implicit in Worrall's Weak UN:

Weak ad hoc-ness: a theory T entailing evidence $e$ is ad hoc with respect to e, if $e$ was used in the construction of $\mathrm{T}$. Then $e$ lends no (or only little) support to $\mathrm{T}$.

Call the view that subscribes to Weak UN and Weak ad hoc-ness heuristic predictivism. Heuristic predictivism invites all sorts of damning objections. First, as many writers have pointed out (Gardner, 1982; Hudson, 2007; Musgrave, 1974), it is highly implausible to assume, as heuristic predictivism does, that contingent and potentially obscure facts about theory construction by individuals should in any way influence the community's assessment of the theory in question. Can we really assume that individuals are normally sufficiently transparent and honest about the way in which they constructed their theories, for the community to be able to make their assessment? Worrall does explicitly claim that to be the case particularly in his study of Fresnel's wave theory of light (1989, p. 154), but even if he were right in this instance, I don't think we can assume that on a general basis. For Worrall's account to be a reasonable account of theory-confirmation, however, this would have to be the case. Otherwise the question of whether a theory is confirmed by the evidence or not regularly would be "up in the air" in the practice of science. And given how difficult it has proven even for philosophers and historians to determine unambiguously, in systematic research, whether or not a certain piece of evidence was used in a theory's construction, it is hard to see that scientists, who do not have a systematic interest in this question, would regularly succeed in answering it. Second, heuristic predictivism is incoherent. Worrall wants to say that there is no asymmetry in the confirmation a theory receives from usenovel accommodations and predictions. For Worrall, use-novel accommodated and predicted evidence are on a par with respect to how much they support a theory. It is this symmetry, after all, that allows him to accommodate the historical evidence surrounding the appraisal of Fresnel's wave theory of light, Einstein's theory of general relativity, and (possibly) Mendeleev's periodic table. But it is questionable whether Worrall is really entitled to that symmetry. After all, temporally novel evidence is by far the best use-novel evidence one 
can get. With temporally novel evidence, one cannot possibly manipulate a theory entailing that evidence so as to accommodate that evidence. In contrast, with evidence $e$ that was already known at the time the theory $T$ entailed it, there is always the possibility that $e$ was used in the construction of T. ${ }^{4}$ Paired with the difficulty of determining equivocally whether or not known evidence was actually used in the construction of a theory or not (as discussed above), temporally novel evidence, even on the heuristic account, is the best evidence one can get. Therefore, it seems that Worrall is not entitled to presume a confirmational symmetry between known-to-be use-novel and not-known-to-be use-novel evidence. ${ }^{5}$ The latter, also on his account, should count more than the former in the appraisal of theories. So if Worrall has shown that the historical record undermines temporal predictivism, that same historical record also undermines heuristic predictivism.

Worrall is well-aware of at least the first difficulty of heuristic predictivism. Indeed, his writings contain a stronger version of the UN criterion, which answers to this first objection (Worrall, 2002, 2005).

Strong UN: Evidence $e$ counts as novel with regard to a theory T entailing e, if $e$ was not needed in the construction of $\mathrm{T}$, or, equivalently, there is no free parameter in $\mathrm{T}$ that would allow us to fix $\mathrm{T}$ in such a way as to accommodate e. Evidence that is novel with regard to T supports $T$.

Accordingly,

Strong ad hoc-ness: a theory T entailing $e$ is ad hoc with respect to $e$ if there is a free parameter in T that needs to be fixed in order for $\mathrm{T}$ to entail $\mathrm{e}$. A theory that is ad hoc with regard to $e$ receives only little or no support from $\mathrm{e}$.

The stronger version circumvents the first problem of heuristic predictivism, since it no longer matters at all whether or not a scientist constructing a theory actually used a certain piece of evidence in the construction of that theory:

[1]t is no part of the heuristic view that it should matter what Einstein was worrying about at the time he produced his theory, what matters is only whether he needed to use some result about Mercury in order to tie down some part of his theory. (Worrall, 1985 p. 319)

[T] here is no specific parameter within that theory that could have been fixed on the basis of [the Mercury observations] so as to produce a specific theory that entailed those observations. (Worrall, 2005 p. 819)

But if all we need to consider, when assessing the evidential support for a theory, is whether that theory does or does not contain a parameter which could have been fixed in order to accommodate certain evidence, then the way in which the theory was constructed can no longer be of interest. Contrary to Worrall's weaker version, the context of discovery and the context of justification now come apart (e.g. Worrall, 2005 p. 819). Given that the actual use of a piece of evidence in the construction of the theory is no longer of interest in this stronger version, the label "heuristic predictivism" no longer seems appropriate. It would therefore perhaps

\footnotetext{
${ }^{4}$ This, in fact, is the rationale that defenders of temporal predictivism often give (see e.g. Lipton, 2004, 140-1).

${ }^{5}$ With known-to-be use-novel evidence I here refer to evidence that was known to be not used in the construction of a theory.
} 
better be referred to as a kind of "parameter-fixing" account. ${ }^{6}$ Worrall's parameter-fixing account not only addresses the first but also the second problem of heuristic predictivism. Whereas heuristic predictivismadditionally to the wanted asymmetry between use-novel predictions and non-use novel predictions-implies an unwanted confirmatory asymmetry between use-novel accommodations and use-novel temporal predictions (apparently without Worrall and others being aware of it), the parameter-fixing account does give Worrall what he needs. On the parameter-fixing account, neither in use-novel accommodations nor in usenovel temporal predictions could we have possibly fixed the parameters of the theory in such a way as to accommodate the data in question: in neither case is there a parameter in the theory that would allow us to do so. Despite the progress Worrall makes with his parameter-fixing account over heuristic predictivism (both of which his writings contain side-by-side without being properly distinguished), ${ }^{7}$ however, even the parameterfixing account faces a number of difficulties. Before discussing those, let us consider another important amendment Worrall makes to Strong ad hoc-ness.

It is an implicit methodological imperative also of Worrall's Strong version of use-novelty that ad hoc modifications be avoided. Sometimes, however, the ad hoc accommodation of evidence is acceptable. This is so when the ad hoc modification of a theory results in independent and supported predictions (Worrall, 2005 p. 818). Schematically, Worrall distinguishes between the 'general' theory $T$ and the 'specific' theory $T$ ' that results from $T$ after the fixing of parameters in T on the basis of evidence $e$ (satisfying Strong ad hoc-ness). Now evidence $e$ does support the general theory T unconditionally if $e$ follows 'naturally' from T. But in this case, there is no parameter in T that needs to be fixed on the basis of $e$ for $T$ to entail $e$ (cf. Worrall, 2002, 2005). There will therefore be no $T^{\prime}$. On the other hand, if there is a free parameter $p$ for evidence $e$ in $T$, there will be a specific version of T with $p$ being fixed on the basis of the evidence e. But then, Worrall has it, $e$ supports $\mathrm{T}^{\prime}$ only conditional on T already being accepted. If there is independent support for T, $e$ is rightly considered to be evidential support for $\mathrm{T}^{\prime}$. Since there is no such independent support for creationism, the Gossified version of creationism, i.e., creationism plus the idea that fossils are the 'playful writings' by God in stone, should not receive any support from the fossil record. In any case, whether there is independent support for T or not, when there is a free parameter in $T$ that needs to be fixed on the basis of $e$ in order to produce $T^{\prime}, e$ does not support T. Worrall also says that the support T' receives from $e$ does not 'spill over' to T. However there is one important exception. If T' makes a (use-novel) prediction $e^{\prime}$ (not predicted by $\mathrm{T}$ ), which then gets confirmed, $e^{\prime}$ will support T. In other words, in that case the support $\mathrm{T}$ ' receives from $e^{\prime}$ does 'spill over' to T. Now recall that $T^{\prime}$ was the outcome of fixing a free parameter on the basis of evidence $e$, which by Worrall's lights prima facie qualifies as an ad hoc accommodation. Although $e$ was accommodated in an ad hoc fashion, and therefore strictly speaking does not support $\mathrm{T}$, this is rendered methodologically kosher retrospectively by the independent support that the fixing of parameters in T generated. Worrall illustrates this scheme with an intuitive example. When Adams and Leverrier used the data of Uranus to 'adjust' the Newtonian theory so that it would entail the right orbit for Uranus (essentially, by adjusting the assumption about the number of planets in our solar system), the 'new' theoretical system (i.e., Newton's theory plus adjusted auxiliary of planet

\footnotetext{
${ }^{6}$ Hitchcock and Sober (2004) also develop a parameter-fixing account. I will discuss their account in the next section.

${ }^{7}$ Indeed, the two unacknowledged versions of Worrall's account have caused a fair amount of confusion in the literature. See e.g. the exchange between Barnes (2005) and Worrall (2005).
} 
number) generated a prediction (the existence of the planet Neptune) which was subsequently confirmed. Worrall concludes that, due to the independent support that ensued, "the use of that data [of Uranus] in the construction of that theoretical system cannot be a "bad thing", although "good book-keeping" requires us to say that Uranus data do not support Newton's theory (Worrall, 2002 197). Consider Worrall's slightly more complicated example of Fresnel's wave theory of light. Fresnel's 'general' theory of light describes the 'functional relationship' between the variables of wavelength (W) and measurable slit and fringe distances (SF) in the two-slit experiment. The general theory does not specify why a particular W should be measured given a particular monochromatic light source. A particular light source's W, however, can be determined by performing the relevant two-slit experiment and by fixing SF accordingly. The theory resulting from this parameter-fixing Worrall, again, refers to as the 'specific' wave theory. Although Strong ad hoc-ness is thus satisfied, the (apparent?) ad hoc accommodation of the data from a monochromatic light source such as sodium arc is legitimate, because-in accord with Worrall's independent support condition-the specific wave theory generates predictions that are independently supported (e.g. fringe separations in other monochromatic light experiments). The support that those data lend to the specific theory does 'spill over' to the general theory. But still, in accord with Worrall's good book-keeping, the data that were used to fix the parameter $\mathrm{W}$ in the general wave theory in order to generate the specific wave theory do not lend any support to the general theory.

The above amendment of Worrall's account has provoked criticism. First, Barnes (2005) criticizes Worrall for the idea that the evidence which needs to used in the construction of a theory should not support that theory. ${ }^{8}$ Consider the following example. In the standard model in particle physics more than twenty free parameters need to be fixed on the basis of experiments for the theory to be empirically accurate. It would be clearly odd to say that evidence that needed to be used to fix the parameters of the standard model does not support the standard model (or more precisely, that the support the 'specific' version of the model receives from that evidence does not 'spread' to the 'general' model). If that were so, the standard model would have rather meager support indeed. Second, as Barnes (2005) and also Hudson (2007) point out, Worrall's independent support condition on "acceptable" ad hoc moves is flawed. For instance, creationists who propose to view a particular fossil record as the 'playful writings' by God in stone (referred to by Worrall as the 'Gosse dodge') will see their 'theory' being independently confirmed by further findings of fossils around the world. But, of course, the support the 'specific' creationist theory (i.e., the Gossified version) would receive from such independent confirmation, contra Worrall, should not spread to the 'general' creationist theory. Creationism is a spurious theory with or without independent support for the Gossified version. ${ }^{9}$ Another, perhaps slightly more convincing example is the following. The Lorentz-Fitzgerald contraction hypothesis (LFC) is one of the prime examples for an ad hoc hypothesis. LFC was invoked to accommodate-in dynamical terms - the nullresult of the Michelson-Morley's ether drift experiments (1887). Popper (1959) sought to dismiss LFC as ad hoc

\footnotetext{
${ }^{8}$ Worrall (2005 820) has rejected this critique due to qualms with specifics of Barnes's example (parameter-fixing in Fresnel's theory).

${ }^{9}$ Worrall (2005), in response to Barnes (2005), seems to admit that much when he introduces the additional requirement that there be "logical constraints imposed by the general theory". These constraints, present in the wave theory of light but not in creationism, are "integral part" of an independent prediction counting in favour of the general theory (as in the wave theory) (822). Although Worrall illustrates these "logical constraints", he fails to spell them out conceptually.
} 
modification for its failure to generate any independently testable predictions (Popper's demands were thus weaker than Worrall's). Contra Popper, Grünbaum (1959) pointed out that LFC did make independent predictions. It for instance did (falsely) predict a non-zero degree of ether drift. Although this prediction was not detectable with the Michelson-Morley experiments, it should have been detectable in a variation of those experiments, namely the so-called Kennedy-Thorndyke experiments (cf. e.g. Janssen, 2002). Now suppose that the independent prediction by LFC had been confirmed in those latter experiments. Then, according to Worrall's scheme, that successful independent prediction would have supported the theory that LFC was supposed to save, namely Lorentz' ether theory. But is it reasonable to assume that? I think not. Lorentz's theory plus LFC was spurious, no matter whether or not that combination was capable of generating independent support. What is wrong with theories like Lorentz', I surmise, is not so much their need for using certain data to fix their parameters, but rather their lack of coherence. I shall spell out the details of this view in Section 4 of this paper. But before doing so, I shall add insult to injury by arguing that, contrary to Scerri and Worrall (2001), temporal predictivism is indeed supported by the historical facts surrounding the appraisal of Mendeleev's periodic table.

\section{The appraisal of Mendeleev's periodic table - the evidence}

The controversy about the status of Mendeleev's successful predictions of new chemical elements has centered around two pieces of evidence. The first piece regards the award of the Davy medal to Mendeleev and the second piece concerns bibliometric data (Brush, 1996). Let us briefly consider them in turn.

Maher (1988), Lipton (2004), and Barnes (2008) have cited the award of the Davy medal to Mendeleev in 1882 by the Royal Society as evidence for temporal predictivism. By the time the medal was awarded, two chemical elements (gallium and scandium) had been discovered in a period of 15 years, which Mendeleev had predicted when first proposing his periodic table (1871). Maher, Lipton, and Barnes seem to treat the fact that the Davy medal was not awarded to Mendeleev before any predicted elements were discovered as evidence for predictions playing a vital role in the appraisal of Mendeleev's table. Yet this alleged support for temporal predictivism can quickly be dismissed. As Scerri and Worrall (2001) point out, there is no mention whatsoever in the Davy medal laudation of Mendeleev's successful predictions of chemical new elements. Rather, emphasis is put on the "marvelous regularity" in the properties in two series of already known elements as revealed by Mendeleev's periodic table. Furthermore, the Davy medal was jointly awarded to Lothar Meyer for his table of chemical elements, who, contrary to Mendeleev, did not make any predictions about any new elements.

The second piece of evidence temporal predictivists cite in support of their view is the fact that Mendeleev's periodic table received a real boost of citations in the period of 1876-1885, i.e., shortly after the first successful discovery of one of Mendeleev's predicted chemical elements in 1875 (Barnes 2008). In that period the number of citations in fact tripled compared to the number of citations Mendeleev's periodic table received in the first four years after its publication (and before the confirmation of one of his predictions). Could there be any clearer evidence for temporal predictivism than that? But a closer look at the bibliometric data sheds some doubt on this. In the years in which gallium (1875), scandium (1879), and germanium (1886) 
were discovered, the respective citations of Mendeleev's periodic table which also mentioned the newly discovered elements were 15 out of 27,13 out of 21 , and 7 out of 26, respectively. Obviously a large number of those authors who discussed Mendeleev's periodic table (in fact the majority of 53\%) did not refer to the successful predictions shortly after the respective elements were discovered. With Scerri and Worrall one may wonder that "it would be strange indeed for an author who had been drawn to Mendeleev's scheme in large part by its predictive success to make no mention of that success at all” (p. 433). Thus, the abovementioned evidence for temporal predictivism in the case of Mendeleev's successful prediction of new chemical elements is at best equivocal.

In addition to these two pieces of evidence, however, there is further historical material that needs to be considered in the assessment of the status of novel predictions for the acceptance of Mendeleev's periodic table. This material concerns Mendeleev's contrapredictions.

\subsection{Contrapredictions}

Successful contrapredictions (a term coined by the historian Stephen Brush) are predictions of theories that induce corrections to empirical results that were hitherto accepted as correct. Interestingly Mendeleev himself highlighted contrapredictions as of utmost importance in the assessment of the periodic table:

Where, then, lies the secret of the special importance which has since been attached to the periodic law, and has raised it to the position of a generalisation which has already given to chemistry unexpected aid, and which promises to be far more fruitful in the future and to impress upon several branches of chemical research a peculiar and original stamp? [...] In the first place we have the circumstance that, as soon as the law made its appearance, it demanded a revision of many facts which were considered by chemists as fully established by existing experience. (1901 p. 475)

In Mendeleev's time, the atomic weight of an element was determined by the product of an element's valence and its empirically-determined "equivalent weight" ${ }^{10}$.Accordingly, there were two sorts of contrapredictions Mendeleev made: (i) predictions that demanded changes to an element's presumed valency and (ii) predictions that demanded changes to an element's presumed equivalent weight. Mendeleev made several counterpredictions of both (i) and (ii), whereby most proposed atomic weight changes associated with (i) turned out to be correct, and most proposed changes associated with (ii) incorrect(Smith, 1976 p. 326ff.) ${ }^{11}$. There were three principles on the basis of which Mendeleev made his contrapredictions (and in fact also his novel predictions): atomic weight ordering from lower to higher values, family resemblance of the elements in a particular group in the table, and "single occupancy" of places in the table. Any discrepancy in the results that the application of each of those principles implied, for Mendeleev, pointed to the re-assessment of atomic weight, family resemblance, or both. Mendeleev never was prepared to violate the principle of atomic-weight ordering, despite genuine exceptions to it. ${ }^{12}$ Whereas before mid-1869 Mendeleev mostly re-positioned

\footnotetext{
${ }^{10}$ An element's equivalent weight is its relative weight as determined by the proportion with which it combines with hydrogen (and later oxygen).

${ }^{11}$ With regard to the "placing" of the elements in the periodic table, however, the picture was reversed (cf. Smith, $1976 \mathrm{p}$. 326ff.).

${ }^{12}$ These exceptions concern so-called 'pair reversals', i.e. cases in which an element E1, on the basis of its chemical properties, would be grouped after another element E2, despite E1 having a lower weight than E2. For instance,
} 
elements in the table while retaining the weight, after mid-1869 most of his re-positioning involved a change in atomic weight (ibid.). Let us briefly consider three examples for Mendeleev's successful contrapredictions.

Mendeleev's perhaps boldest contraprediction concerns uranium. Uranium was first isolated in 1841 and was usually assumed to have an atomic weight of 120 and a valence of 2 . But with this weight one of the constraints of Mendeleev's periodic table (continuous decrease of valence from group IV to group VII) would have been violated, since uranium would then have to be placed in between the tetravalent tin (122) and trivalent antimony (118). Mendeleev proposed that the atomic weight for uranium be 240 by doubling its assumed valence. In a later edition of the Principles of Chemistry Mendeleev wrote that "uranium ... has played a prominent role in the confirmation of the periodic law, because with the recognition of this law a change in its atomic weight was called for, and was proved valid". For Mendeleev, this served "as convincing evidence of the generality of the periodic law" (cited in Smith, 1976 p. 335, added emphasis).

Mendeleev also suggested a correction to beryllium, which, on the basis of a number of experiments had been determined to be trivalent and as possessing an atomic weight of about 13.5-14 (cf. Scerri, $2007 \mathrm{p}$. 128). Again, according to Mendeleev (1901) this weight "became generally adopted and seemed to be well established" (p. 484). However, "there was no place in the system for an element like beryllium having an atomic weight of 13.5" (ibid). The chemical properties of beryllium were just too dissimilar to nitrogen (atomic weight of 14) to place it next to it. Instead Mendeleev surmised that beryllium was bivalent, because it could then be placed in between lithium (7) and boron (11) in group II. After "a divergence of opinion [which] lasted for years" (ibid.), Mendeleev was vindicated in 1889.

Ironically the successful prediction of gallium, the first discovered element to confirm any of Mendeleev's novel predictions, is in fact a partial contraprediction. In 1875, apparently without being aware of Mendeleev's prediction, the French chemist Lecoq de Boisbaudran discovered gallium. He determined gallium's density as $4.7 \mathrm{~g} / \mathrm{cm}^{3}$. Mendeleev, however, had predicted a significantly higher value, namely about $6.0 \mathrm{~g} / \mathrm{cm}^{3}$. Only after becoming aware of that contraprediction, Boisbaudran realized that his original measurements had been contaminated with metallic sodium (which he used as a reducing agent in the isolation of free gallium). And indeed, Boisbaudran now gained a density of $5.935 \mathrm{~g} / \mathrm{cm}^{3}$, very much in agreement with Mendeleev's contraprediction. Later Mendeleev claimed that without the periodic table "nothing would have pointed to the incorrectness of this [earlier] determination, nothing would have prompted the verification of the difficulty obtained and separated gallium" (1901, p. 262).The successful prediction of gallium being a partial contraprediction (about some of the properties of gallium) would be particularly significant insofar as contrapredictions are considered to be a different sort of beast from novel predictions. Because then, contrary to the temporal predictivists, the boost in citations to Mendeleev's periodic table (see above) may in fact not be due to the successful temporal prediction of gallium but rather due to the

Mendeleev predicted a value of 125 for tellurium rather than the previously accepted value of 128 so that it could be placed before iodine (127). And indeed chemists tried to verify this prediction and experimentally re-determined the weight of tellurium as approximately 124-126, i.e. below the value of iodine. These values were however later deemed erroneous; iodine indeed comes before tellurium in the periodic table-despite its higher weight. This apparent anomaly would ultimately be eliminated by changing the main ordering criterion from atomic weight to atomic number. 
successful contraprediction of gallium's properties. But are contrapredictions really significantly different from temporally novel predictions?

I believe (with Brush (1996), but contrary Scerri (2007)) that we should treat contrapredictions as a particular form of temporally novel predictions. Just like in standard temporally novel predictions, and contrary to accommodations, in contrapredictions it is not known at the time a particular prediction is being made whether the contraprediction will turn out to be correct. Contrapredictions and temporally novel predictions differ of course in that in the former there is, and in the latter there is not, data relevant to the assessment of the prediction. But I don't think that this is a significant difference. This judgment is in fact supported by Mendeleev's own writings. With regard to the successful contraprediction of the weight of beryllium Mendeleev noted that he took it "as important in the history of the periodic law as the discovery of scandium" (e.g. 1901 p. 485). ${ }^{13}$ Indeed Mendeleev, when commenting on the reasons for the widespread acceptance of the periodic table, highlighted contrapredictions and temporal predictions (e.g. 1901 p. 231). Assuming, reasonably I think, that Mendeleev was not deluded about the reason for the success of his scheme, it seems to be quite clear that predictions did indeed enjoy a special status in the appraisal of Mendeleev's periodic table-contrary to what Scerri and Worrall (2001) claim. This is consistent with bibliometric data considered above. ${ }^{14}$ But even though the appraisal of the periodic table indeed seems to support temporal predictivism, temporal predictivism is still at odds with the following facts: (i) the absence of any mention of Mendeleev's successful predictions in the Davy medal laudation, and (ii) the appraisals of Fresnel's wave theory and Einstein's general theory of relativity, for the former of which Worrall (1989) has made a good case that temporally novel predictions were not valued higher than accommodation in the community and for the latter of which Brush (1994) has made the same claim (see Introduction). How are these facts to be reconciled? In the remainder of this paper I shall try to develop an answer to (i). A possible answer to (ii) will be sketched at the end of this essay.

\section{Local-symptomatic predictivism}

Hitchcock and Sober (2004) introduce various useful distinctions, which l'd like to introduce before making my own proposal about the role and function of successful predictions. First of all, Hitchcock and Sober distinguish between a weak and a strong form of temporal predictivism. (For the sake of simplicity I shall from now on refer to temporal predictivism merely as predictivism, and to temporally novel predictions merely as predictions). According to the strong form of predictivism, predictions are (for whatever reason) intrinsically more valuable than accommodations. According to the weak form, predictions are more valuable than accommodations only because they are signs of another theoretical property which may not be directly accessible to scientists. Let me refer to the latter form as symptomatic predictivism. Another distinction Hitchcock and Sober introduce is the distinction between global and local predictivism. According to global predictivism, predictions count more than accommodations in all possible contexts. According to local predictivism, predictions count more than accommodations only in particular contexts.

\footnotetext{
${ }^{13}$ Contrapredictions do not only occur in the context of the periodic table. See Schindler (2013).

${ }^{14}$ Note that those data do not contain any information on Mendeleev's contrapredictions.
} 
In Hitchcock and Sober's local and symptomatic predictivism, predictions can, but need not be, more valuable than accommodations in the appraisal of theories. Predictions are more valuable than accommodations in theory appraisal, if it is not obvious whether or not a theory has over-fitted the data, whereby an overfit of the data consists in the theory's accommodation of data it should have not accommodated (because these data are just due to the 'noise' in the experiment). Overfitting, in turn, reduces the theory's capacity to accurately predict new data. Thus, successful novel predictions can serve as an indicator for a theory not being overfitted. This is why Hitchcock and Sober's form of predictivism is symptomatic. Hitchcock and Sober's predictivism is local because a theory's predictions are only valuable in contexts in which it is not clear that the theory was not overfitted. In the case of Fresnel, Hitchcock and Sober hold, it was clear that the theory was not overfitted to the data and therefore the white spot prediction was not needed for evaluating Fresnel's theory as being a good theory (p. 29).

Prima facie, Hitchock and Sober's local and symptomatic predictivism appears to be a plausible account also of Mendeleev's periodic table. When Mendeleev first proposed his periodic table there was a risk, as for anyone proposing a classification of chemical elements at the time, that he had overfitted the data, i.e. that he had accommodated empirical information about chemical elements that was wrong. It was only after his successful temporally novel predictions and his successful contra-predictions that this was shown not to be the case. When it comes to the details of the application of Hitchock and Sober's account to the case of Mendeleev's periodic table, however, things start to look less pretty.

On Hitchcock and Sober's account the rationale for a model being more predictive of new data when it is less overfitted to the current data has to do with the number of parameters of a model. The fewer parameters a model possesses, the less likely it is to be tied down to a particular data set. The fewer parameters the model has, the more "flexible" it is in accommodating new data sets that share a certain "trend" with the previous data sets, but differ in the exact distribution of data points. Hence overfitting is directly related to the number of parameters: the fewer parameters, the simpler the model, the less likely the overfit, and the more likely the accommodation of future data. It is important to stress that the parameters Hitchcock and Sober are talking about are empirical parameters. ${ }^{15}$

It is not easy to see how this story behind Hitchcock and Sober's local and symptomatic predictivism could be applied to Mendeelev's periodic table. The only empirical parameters that Mendeleev was able to fix on the basis of the evidence were the slots for the chemical elements in the periodic table. But then, the number of parameters seems to make little difference to whether or not Mendeleev's table overfitted the data. Had Mendeleev accommodated the false data, his table would have had just as many parameters as it actually did have without overfitting the data. Perhaps Hitchcock and Sober could discern other sorts of parameters in Mendeleev's table for which they could make their case. But it is unclear what sorts of parameters that could be. Assuming that a local and symptomatic form of predictivism is the right kind of theory to describe Mendeleev's table, it appears that a new rationale for this brand of predictivism is needed. In the next section I will try to develop one.

\footnotetext{
${ }^{15}$ In the Fresnel example, the „only“ parameter that Fresnel's theory contained was the "wavelength of the light used in the experiment" (Hitchcock \& Sober, 2004 p. 29).
} 


\subsection{Coherence}

Suppose someone proposes a theory that stands in contradiction with some of the relevant facts. Why should you give that theory any credit let alone adopt it? This question must also be posed for Mendeleev's periodic table. Why was it taken seriously rather than being rejected out of hand? After all, it too made predictions in contradiction with apparently established facts (see Section 3.1). Clearly, a theory that stands in contradiction with some of the known facts must have a property that makes its adoption attractive despite its lack of empirical fit. In this section I want to argue that in the case of Mendeleev's table, this property concerned its coherence.

In order to be able judge whether Mendeleev's periodic table was a coherent classification, we need to know what it is for a theory to be coherent. This is a difficult question and it is not clear that there exists a satisfactory philosophical answer. Usually, coherence is described as the property of "how well things hang together". Rather than trying to give necessary and sufficient conditions for coherence, I would like to illustrate this property with two examples ${ }^{16}$.

The first example concerns the explanation of the Michelson-Morley (MM) aether drift null result. Two explanations were invoked to explain the result. The first came from the ether theory, which was amended with the notorious Lorentz-FitzGerald contraction hypothesis (LFC). The second explanation of the MM result was of course Einstein's special theory of relativity. Whereas in the amended aether theory there remained "a strict separation of ether and matter", Einstein's theory was able to provide a coherent theory, in which the laws governing matter and fields received a common justification in terms of Minkowski spacetime (cf.Janssen, 2002). The second example concerns the contrast between the Copernican and the Ptolemaic system. Although the ad hoc-ness of the Ptolemaic system is often associated with its use of epicycles, the latter cannot account for the ad hoc-non-ad hoc contrast between the two theories. Copernicus never complained about the use of epicycles by the Ptolemaists and he in fact used the device himself rather extensively (Kuhn, 1957). However, Copernicus did complain about other devices, such as the equant point, which violated Copernicus's "first principle" of uniform circular motion. Perhaps more importantly, Copernicus also famously compared Ptolemaic astronomy to incoherent sculpturing, because it constructed its models on a planet by planet basis without any concern for how the models might fit together: "it is as though an artist were to gather the hands, feet, head and other members for his images from diverse models, each part excellently drawn, but not related to a single body, and since they in no way match each other, the result would be a monster rather than a man" (Copernicus, 1992 my emphasis). ${ }^{17}$

\footnotetext{
${ }^{16}$ There have been recent attempts to make the notion of coherence precise within the framework of Bayesianism (Bovens \& Hartmann, 2003). According to them, "[c]oherence is a property of an information set that boosts our confidence that its content is true ceteris paribus when we receive information from independent and partially reliable sources". However, not only would it lead us too far astray to grapple with the details of their account, but it also not clear that their framework could shed any interesting light on the discussion here.

${ }^{17}$ It is interesting to note about these two examples that a theory's lack of coherence and the scientific community's judgment about it being ad hoc seem to be closely related.
} 
In analogy to these informal illustrations of the theoretical property of coherence I want to suggest that Mendeleev's classification was coherent because it constituted the first serious attempt in history to relate all elements and their chemical properties to a single ordering criterion, namely to atomic weights. Although previous attempts to order the elements did identify periodicities, they never went as far as subordinate all elements to the atomic weight criterion (cf. Smith, 1976).Mendeleev's periodic table, in his own words, "proclaims loudly that the nature of the elements depends above all on their mass" (e.g. 1901 p. 231). However, at the time Mendeleev proposed his table it was not clear whether his insistence on a coherent ordering of the elements was not too optimistic. After all, his coherent classification was at odds with some empirical information about the chemical elements and previous classification attempts had all failed to come up with a coherent ordering. Only after some of Mendeleev's contrapredictions turned out to be correct, did the confidence in the correctness of Mendeleev's scheme receive a boost. But again, without the prior attraction of Mendeleev's classification, which, I suggested, sprung from its coherence, Mendeleev's scheme might have been rejected out of hand rather than leading the way towards the correction of apparently established knowledge. I therefore conclude that, when coherence is the driving force behind the initial adoption of a theory ${ }^{18}$ (despite contrary evidence), successful (contra-)predictions are important indicators for the coherence of facts identified by the theory to be real. In other words, the study of the appraisal of Mendeleev's periodic table suggests a form of symptomatic predictivism according to which successful predictions count more than accommodations in the appraisal of theories if they reveal the coherence identified by the theory as being real.

In the form of symptomatic predictivism I propose, scientists generally suspect coherence to be a truthconducive theoretical virtue. This is why coherent theories are not rejected out of hand even if they stand in contradiction with some of the known evidence (as did Mendeleev's periodic table). But of course, there is no guarantee that coherent theories will really turn out to be correct. This is why successful contrapredictions are important in the appraisal of theories: they confirm the scientist's suspicion that coherence might be a truthconducive property in the case at hand.

It is interesting to note that my form of symptomatic predictivism is considerably weaker than Hitchcock and Sober's form of symptomatic predictivism. Contrary to their form, my form of symptomatic predictivism does not stipulate a necessary connection between a theory's property of being coherent and its capacity to produce successful predictions. Whether a theory produces successful predictions will simply depend on whether or not the coherence of facts identified by the theory is real.

One may wonder about other sub-types of the form of symptomatic predictivism proposed here. That is, one might wonder whether theoretical virtues than coherence (e.g. simplicity, unifying power, fertility), may play a similar role as coherence does in the form of predictivism proposed here. In principle, it would seem,

\footnotetext{
${ }^{18}$ Note that it is not at all implausible to treat Mendeleev's periodic table as a theory, for it is (minimally) explanatory of a certain set of facts. The membership in a certain chemical group, as delineated by the periodic table, explains the apparent coincidence that certain chemical elements that differ considerably in their atomic weights possess similar chemical properties. Since successful predictions were made on the basis of the periodic table, the periodic table clearly satisfies two central characteristics of what it is for something to be a theory.
} 
such other forms may very well exist. But of course they would have to be shown to exist, a task which is beyond this essay. ${ }^{19}$

\section{Conclusion}

With regard to the appraisal of Mendeleev's periodic table of chemical elements I argued in this paper for a form of symptomatic (temporal) predictivism according to which a theory's predictions are indicative of the coherence of facts being identified by the theory being real. Thus, Mendeleev's successful predictions were only of import in the appraisal of Mendeleev's periodic table insofar they showed that Mendeleev's idea of relating all chemical elements to the single ordering criterion of atomic weight was approximately correct. How these conclusions are to be reconciled with the facts about the appraisal of Fresnel's wave theory of light and Einstein's general theory of relativity will have to await further clarification. But it may be suggested tentatively that the right general view of the status of predictions in the appraisal of theories in science is a localsymptomatic predictivism. That is, in some contexts, scientists apparently do not need to await a theory's successful predictions in order to judge that the theory in question has identified a real (coherent) relationship. Perhaps there is a slight asymmetry between contrapredictions and novel predictions after all: showing that the theory's contradiction with the facts was only apparent might be a more pressing demand in deciding that the relations identified by a theory are likely to be real than checking whether the theory might possibly be contradicted by facts that have not been gathered yet.

\section{Acknowledgments}

I thank Eric Barnes, Helge Kragh, and two anonymous referees for reading drafts of this paper and for providing input that allowed me to make a number of important improvements. All remaining mistakes are of course mine. I also thank the participants of the workshop on Novel Predictions at the University of Düsseldorf in March 2011 for helpful comments. I am grateful to the Zukunftskolleg at the University of Konstanz and the German Research Foundation (DFG) for financially supporting part of the research that underlies this paper.

\section{References}

Barnes, E.C. (2005). On Mendeleev's predictions: comment on Scerri and Worrall. Studies in History and Philosophy of Science Part A, 36(4), 801-812.

Barnes, E.C. (2008). The paradox of predictivism. Cambridge: Cambridge University Press.

Bovens, L., \& Hartmann, S. (2003). Solving the riddle of coherence. Mind, 112(448), 601-633.

Brush, S.G. (1994). Dynamics of Theory Change: The Role of Predictions. PSA: Proceedings of the Biennial Meeting of the Philosophy of Science Association, 133-145.

Brush, S.G. (1996). The reception of Mendeleev's periodic law in America and Britain. Isis, 87(4), 595-628.

Copernicus, N. (1992). On the Revolutions (translation and commentary by Edward Rosen). Baltimore: Johns Hopkins University Press.

Gardner, M.R. (1982). Predicting novel facts. British Journal for the Philosophy of Science, 1-15.

${ }^{19}$ But see Schindler (2013). 
Grünbaum, A. (1959). The falsifiability of the Lorentz-Fitzgerald contraction hypothesis. The British journal for the philosophy of science, 10(37), 48-50.

Harker, D. (2006). Accommodation and prediction: The case of the persistent head. The British journal for the philosophy of science, 57(2), 309-321.

Hitchcock, C. , \& Sober, E. (2004). Prediction versus accommodation and the risk of overfitting. The British journal for the philosophy of science, 55(1), 1-34.

Hudson, R.G. (2007). What's Really at Issue with Novel Predictions? Synthese, 155(1), 1-20.

Janssen, M. (2002). Reconsidering a scientific revolution: The case of Einstein versus Lorentz. Physics in Perspective, 4(4), 421-446.

Kuhn, T.S. (1957). The Copernican revolution: planetary astronomy in the development of western thought. Harvard: Harvard University Press.

Lange, M. (2001). The apparent superiority of prediction to accommodation as a side effect: a reply to Maher. The British journal for the philosophy of science, 52(3), 575-588.

Lipton, P. (2004). Inference to the best explanation. London: Routledge.

Maher, P. (1988). Prediction, accommodation, and the logic of discovery. Paper presented at the PSA: Proceedings of the Biennial Meeting of the Philosophy of Science Association.

Mayo, D.G. (1991). Novel evidence and severe tests. Philosophy of science, 523-552.

Mendeleev, D. (1901). The principles of chemistry [tr. from the Russian (sixth edition) by George Kamensky; ed. by A.J. Greenaway]. New York Collier.

Musgrave, A. (1974). Logical versus historical theories of confirmation. British Journal for the Philosophy of Science, 1-23.

Popper, K.R. (1959). The logic of scientific discovery. London: Routledge.

Scerri, E.R. (2007). The periodic table: its story and its significance. Oxford: Oxford University Press.

Scerri, E.R., \& Worrall, J. (2001). Prediction and the periodic table. Studies in History and Philosophy of Science Part A, 32(3), 407-452.

Schindler, S. (2013). Theory-laden experimentation. Studies in History and Philosophy of Science Part A, 44(1), $89-$.

Smith, J. R. (1976). Persistence and Periodicity: A Study of Mendelcev's Contribution to the Foundations of Chemistry. (Ph.D.), University of London.

White, R. (2003). The epistemic advantage of prediction over accommodation. Mind, 112(448), 653-683.

Worrall, J. (1985). Scientific discovery and theory-confirmation. Change and progress in modern science, 301331.

Worrall, J. (1989). Fresnel, Poisson and the 'White Spot': The Role of Successful Prediction in Theoryacceptance: The Uses of Experiment, Cambridge: Cambridge University Press.

Worrall, J. (2002). New evidence for old. In P. Gardenfors (Ed.), In the Scope of Logic, Methodology and Philosophy of Science (pp. 191-209). Dordrecht: Kluwer

Worrall, J. (2005). Prediction and the 'periodic law': a rejoinder to Barnes. Studies in History and Philosophy of Science Part A, 36(4), 817-826. 